Notfall Rettungsmed 2011 · 14:177-177

DOI 10.1007/s10049-011-1453-9

Online publiziert: 14. April 2011

(c) Springer-Verlag 2011

\author{
H.-R. Arntz ${ }^{1}$ - U. Kreimeier ${ }^{2}$ \\ ${ }^{1}$ Medizinische Klinik II, Kardiologie und Pulmonologie, Charité, \\ Campus Benjamin Franklin, Berlin \\ ${ }^{2}$ Klinik für Anaesthesiologie, Klinikum der Universität München
}

\title{
Strukturierte Abstracts: ein neues Layout bei Notfall + Rettungsmedizin
}

Notfall + Rettungsmedizin hat sich zum Ziel gesetzt, seine Leserinnen und Leser mit Übersichten, Originalien, der CME-Weiterbildung und anderen hochwertigen Beiträgen in die Lage zu versetzen, sich wissenschaftlich und praktisch auf dem neuesten Stand der Erkenntnisse auf dem Gebiet der Notfallmedizin zu bewegen. Neben frei eingereichten Beiträgen (Originalien, Kasuistiken) erscheinen in jedem Heft 3-5 zu einem „Leitthema“ gebündelte Beiträge, die als State of the Art Reviews oder Übersichtsarbeiten jeweils einen Themenbereich aus verschiedenen Blickwinkeln beleuchten. In dieser Zusammenstellung nimmt diese Zeitschrift eine gewisse Sonderstellung ein.

Abstracts stellen die inhaltliche Kurzfassung eines Beitrags dar. Sie sollen ein Maximum an Information mit einem Minimum an Wörtern beinhalten. Vorbei ist die Zeit, als bei der Einreichung von Abstracts zur Anmeldung von Beiträgen für wissenschaftliche Tagungen die Fontgröße verändert wurde, um möglichst viele „Anschläge “ unterzubringen. Die Vorgaben heute lauten durchweg: „Der Abstracttext darf nicht mehr als... Zeichen inklusive Leerzeichen umfassen." Damit die vorgegebene Kürze des Abstracts trotzdem Garant für die korrekte Wiedergabe des Inhalts ist, hat sich bei der Darstellung von Ergebnissen wissenschaftlicher Untersuchungen die Einteilung in einzelne Abschnitte durchgesetzt. Üblicherweise sind dies

- Hintergrund,

- Ziel der Arbeit (Fragestellung),

- Material und Methode,

- Ergebnisse,

- Schlussfolgerung.

Die CONSORT- (Consolidated-Standardsof-Reporting-Trials-)Gruppe empfahl kürzlich eine quasi Minimalliste essenzieller Punkte, die Autoren bei der Einreichung von Ergebnissen aus kontrollierten klinischen Studien oder Konferenzabstracts einhalten sollten. Hinsichtlich Abstracts wurde ein strukturiertes Format empfohlen [3].

Nicht alle Beiträge in einer Zeitschrift stellen Ergebnisse von Studien dar, manchmal sind es Fragestellungen, die bearbeitet werden und anhand von Metaanalysen dargestellt werden. Gleichwohl war bereits Anfang der 1990er Jahre vorgeschlagen worden, strukturierte Abstracts nicht nur für Originalarbeiten, sondern auch für Übersichtsarbeiten und Leitlinien einzuführen $[1,2]$. Im August 2010 wies knapp ein Viertel aller in die MEDLINE-Datenbank aufgenommenen Abstracts eine derartige abschnittsweise Struktur auf [4].

Strukturierte Abstracts erleichtern die Lesbarkeit und bieten dem Leser den Vorteil, sich schneller orientieren zu können. Sie werden deshalb zukünftig auch in Notfall + Rettungsmedizin von den Autoren ausdrücklich erwünscht und sollen zum Standard in den Rubriken „Leitthema“, „Orginalien - Kasuistiken“ und „Stellungnahmen - Leitlinien“ werden. Wir unterstützen damit das von der U.S. National Library of Medicine (NLM) - National Institutes of Health (NIH) [3] und dem International Committee of Medical Journal Editors (ICMJE) in den „Uniform Requirements for Manuscripts Submitted to Biomedical Journals" (http://www.icmje.org/ manuscript_1prepare.html; online Zugriff am 30.03.2011) dokumentierte und empfohlene Vorgehen bei Einreichung von Beiträgen in medizinischen Fachzeitschriften. Mit der Januarausgabe der von der NLM herausgegebenen Version von MEDlars onLINE (MEDLINE/LMEDLINE) wur- den auch in der bedeutensten medizinischen Wissensdatenbank die Weichen für strukturierte Abstracts gestellt.

Wir haben uns vorgenommen, im Sinne der Qualitätssicherung und Akzeptanz internationaler Standards unsere Autoren zu bitten diesen wichtigen Schritt mit $\mathrm{zu}$ vollziehen und auch Ihnen, liebe Leserinnen und Leser, mit der Einführung von strukturierten Abstracts in eine weitere nicht nur formale, sonder auch inhaltliche Verbesserung in Notfall + Rettungsmedizin zu bieten.

Ihre

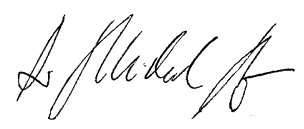

H.-R. Arntz

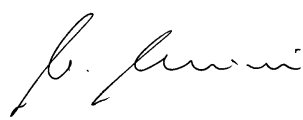

U. Kreimeier

\section{Korrespondenzadresse}

Prof. Dr. U. Kreimeier

Klinik für Anaesthesiologie, Klinikum der Universität München

Nussbaumstraße 20, 80376 München

Uwe.Kreimeier@med.uni-muenchen.de

\section{Literatur}

1. Haynes RB, Mulrow CD, Huth EJ, Altman DG, Gardner MJ (1990) More informative abstracts revisited. Ann Intern Med 113:69-76

2. Hayward RS, Wilson MC, Tunis SR, Bass EB, Rubin HR, Haynes RB (1993) More informative abstracts of articles describing clinical practice guidelines. Ann Intern Med 118:731-737

3. Hopewell S, Clarke M, Moher D, Wager E, Middleton P, Altman DG, Schulz KF (2008) CONSORT for reporting randomized controlled trials in journal and conference abstracts: explanation and elaboration. PLoS Med 5:e20

4. Ripple AM, Mork JG, Knecht LS (2010) Structured abstracts: a neew look for the PubMed(R) abstract display. NLM Tech Bull 375:e11 